\title{
Zehn Grunderkenntnisse für sichere Events
}

1. Personal ist das wichtigste Gut und will wertgeschätzt sein!

2. Veranstaltungen sind dynamisch - Sicherheit muss dementsprechend agil und belastbar sein!

3. Konzepte bilden das Rückgrat jeder Veranstaltung!

4. Fehler lassen sich im gedanklichen Walk Through lokalisieren und bereinigen!

5. Ein objektives und seriöses Risikomanagement ist unerlässlich!

6. Die Kumulation kleiner Einzelprobleme generiert ein Sicherheitsproblem!

7. Problematisch geplante, nicht rund laufende Veranstaltungen verfügen nicht über freie Kapazitäten, mit denen spontan aufkommende Probleme erkannt und adäquat bearbeitet werden können!

8. Die Definition und Wahrnehmung der Rollen und Verantwortungen in der Veranstaltungshierarchie erzeugt eine reibungslose Führung!

9. Nachbetrachtungen von Veranstaltungen liefern die Grundideen für bessere, hochwertigere, sicherere Veranstaltungen in der Zukunft!

10. Sicherheitskultur ist das Ergebnis einer beabsichtigten Entwicklung! 